\title{
Sachwortregister Band 22, 1995
}

Akutes Lungenversagen 298

Alaninaminotransferase 3145

-, Ausschlußwerte 3145

Alloimmunisierung 19

Allosensibilisierung Suppl. 168

Aminosäureanalysen Suppl. 15

Aminosäuren 282

-, Aspartat 282

-, Glutamat 282

Antigen D, D-Kategorie VI 5285

Anti-HCV-ELISA der 3. Generation 3164

Sensitivität 3164

Spezifität 3164 Antikörpersuchtest 1 25,4 232,

Suppl. 1101 Anti-Phospholipid-Antikörper Suppl. 163 Anti-Rh(D)-Therapie 4220

Anzeigepflicht von Arzneimittelrisiken

3 186

Aprotinin Suppl. 174

Autologe Transfusion Suppl. 1139

Autologes gewaschenes Erythrozytenkon-

zentrat Suppl. 1137 - Retransfusionsmodell Suppl. 127

Blockade des retikuloendothelialen Systems

220 Blutgasanalysen 298 Blutgas-Monitoring 298 Blutgruppen Suppl. 1130 -, seltene 5285

Blutgruppensysteme: ABO, Rhesus, Kell

285

Blutkomponenten, Nebenwirkungen 3186 Blutkomponententherapie Suppl. 1130

Blutkonservierung Suppl. 136 Blutplasma 5274 Blutplättchen 136 Blutpräparate Suppl. 186 Blutprodukte 4258 Blutspende 3 175, Suppl. 1 130,

Suppl. 1139 -, Aufwandsentschädigung 119 Blutspender 3 145,4 226, Suppl. 189

Blutstammzellen Suppl. 1 116,

Suppl. 1119 Bluttransfusion 2 91,4 208, Suppl. 1130 -, Erythrozyten 291 -, Vorhersage 291

Blutverlust Suppl. 174 Blutvolumen 274

C.A.T.S ${ }^{\circledR}$ Suppl. 1137 CD34+-Zellanalyse 3152 CD34+-Zellen Suppl. 1125

Chargendokumentation 4258 Cholesterinscreening 4226

Computergestützte Verfahren bei Herstel-lung von Blutprodukten 3196

Qualitätssicherungssystem 3196 Softwareprüfung 3196 Validierung der Blutgruppenbe-

stimmung 3196

Concept Autologe Transfusion (CAT) Suppl. 1137

CR1 5310

Dauerblutspender Suppl. 1145 Deglycerolisation Suppl. 130 Dextran 6332 Dipeptide 5317 
Direkter Antiglobulintest 1 31, 2 117,4 232 Durchflußzytofluorometrie 6344

Durchflußzytometrie 3159 -, multiparametrische 3152 Durchflußzytometrische Analyse Suppl. 1125

EDV 4258 EIA 4208 -, Spezifität 4208 Eigenansatz 4232 Eigenblut Suppl. 130

Eigenblutspende Suppl. 1133 -, Information fürÄrzte Suppl. 1133 -, Information für Patienten Suppl. 1133 -, Organisation Suppl. 1133 Einfrieren Suppl. 136 Eingefrorene Zellen 3152

Eisenmangel Suppl. 1 142, Suppl. 1145 Eisenstatus Suppl. 1145 Eisenstoffwechsel Suppl. 1139

Eisensubstitution Suppl. 1145 Eisentherapie Suppl. 1139 Eisenüberladung Suppl. 1142

Elastase 3159 Elektrophoresen Suppl. 15 Enterale Ernährung 5280 Epidemiologie 5285

Ernährungsparameter 5280 Erstblutspender Suppl. 1142 Erythropoetin Suppl. 1139 Erythrozyt 5310

Erythrozytäre Antigene und Antikörper 6344

Antikörper 4232 Erythrozyten Suppl. 127 Erythrozytenkonzentrate 6355 Erythrozytenvolumen

274 Extrakorporale Zirkulation 282

-, Blutkardioplegie 282

Faseroptik 298 Festphasentest Suppl. 1101

Fetale Alloimmunthrombozytopenie

Suppl. 177 Fett Suppl. 1137 Fick-Prinzip 6340 Filtration 19

Filtrationseffektivität Suppl. 119 Fließkammersystem Suppl. 1155

G-CSF-Mobilisierung Suppl. 1 116,

Suppl. 1125 Gefrorenes Frischplasma Suppl. 12 ,

Suppl. 121 Geltest 4232

Gelzentrifugationsmethode 125 Gelzentrifugationstest Suppl. 1101 Gerinnung 5296 GLP 3196

Glutamin 5317 Glycerin Suppl. 136 GMP 3196

Hämatokrit 274

Hämatopoetische Progenitorzellen 3152

Hämochromatose Suppl. 1142

Hämodilution 274

Hämolytische Anämie 6344

Hämophilie A, Therapie Suppl. 160

Hämorheologie Suppl. 142

Hämorrhagischer Schock 6355

Harnstoffvernetzte Gelatine 6332

HCV 4208

-, Epidemiologie 4208

HCV-Bestätigungstest Suppl. 189

HCV-Diagnostik Suppl. 189

Hepatitis Suppl. 186

- C 3164

Herzchirurgie 282

-, koronarer Bypass 282

Herzzeitvolumen 6340

HES Suppl. 136

HIV-Diagnostik Suppl. 195

HIV-Screening 3175

-, Kosten 3175 
-, Validität 3175

HLA-nichtident Suppl. 1119

Hospitalmortalität 291

Humanalbumin 6332

Humanes Plasma Suppl. 18

Hydroxyethylstärke 6 332, Suppl. 127

Hyperfibrinolyse 5296

Idiopathischethrombozytopenische

Purpura 4220 IgE Suppl. 114 IgG 136

IgG-Subklassen 6360 Immunadsorption Suppl. 142

Immunglobulin-Handelspräparate 6360 Immunkomplex 5310 Immunmodulation 2110

Immunstatus 5280 Indikationsliste Suppl. 1133 Infektionssicherheit Suppl. 186 Intensivstation

291 Interne Qualitätsüberwachung 6364 Intrakranielle Blutung Suppl. 177

IntrauterineThrombozytentransfusion

Suppl. 177 Intravenöse Immunglobuline 131,

Suppl. 177 In-vitro-Untersuchung 6332 Isoantikörper 131 ISO-Standards Suppl. 1155

Kaskadefiltration Suppl. 142 Katabolie 5317 Katheter Suppl. 1159 Klinische Ernährung 5317

Kohlendioxidpartialdruck 298 Kolloidale Plasmaersatzmittel 6332 Komplement 136,5 310

Korrelation Suppl. 1159 Kryokonservierung Suppl. 1 27, Suppl. 1 30, Suppl. 136

Laborchemische Parameter Suppl. 142 Lagerungscontainer 3152 Lagerungseílekt 5292 LDL-

Apherese Suppl. 142 Lea-Antigen 4249 Leber 2106

Lebertransplantation 5296 Leukozytendepletion 1 9,6 355,

Suppl. 1 19, Suppl. 121 Leukozytenkontamination 4244 Lookback-Untersuchungen Suppl. 189

Lungenchirurgie Suppl. 174 Lupus-Antikoagulans Suppl. 163 Lysozym 3159

Massivtransfusion 5 296,6 355 Metabolismus: Glukose 5274 Metallkorrosion Suppl. 1155

Meßtechniken: Plasmavolumen 5274 Methylenblau Suppl. 1 8, Suppl. Ill Methylenblau/Licht

Suppl. 15 Mikrotiterplatten-Methode 4226 MonoklonaleBlutgruppenreagenzien 2117 -

Reagenzien 4249 Multizentrische Studie 6360

n-3-Fettsäuren 2110 n-3/n-6-Fettsäureverhältnis 2110 n-6-Fettsäuren 2110 Neutrophile

Granulozyten 3159 NichthämolytischeTransfusionsreaktionen Suppl. 114

Operative Infektionen 5280

Papaintest 125 Parenterale Präparate 5317 Parenterales Fett 2110 PBPC-Apherese Suppl. 1125

PCR-Analytik Suppl. 195 PEEP(positive endexpiratory pressure)-

Beatmung 3168 Periphere Blutprogenitorzellen

Suppl. 1125 Phagozytose 3159

Photodynamische Behandlung Suppl. 111 Plasmaersatzmittel 274 Plasmafiltration Suppl. 121

Plasmapherese Suppl. 1130 Plasmaproteine Suppl. 15 Plasmaspende 3175 Plasmavolumen 2

74 Plättchenreaktivität 4237 Polymere Suppl. 1159 Polymermaterialien Suppl. 1155

Produktqualität 4244 Proteinsynthese 2106 Prothetische Implantate Suppl. 1155

Pseudothrombozytopenie 5303

Qualitätskontrolle 4237 -, Blutgruppenserologie 2117 Qualitätssicherung 6 364, Suppl. 186

Qualitätssicherungssystem 6364

RekombinanteFaktor-VIII-Konzentrate

Suppl. 160 Rezeptoren 136 RIBA 4208

Risikoscore Suppl. 1133 Röhrchentest Suppl. 1101 
Sauerstoffpartialdruck 298 Sauerstoffsättigung 6340 Score-Systeme 291 -, Acute Physiology and Chronic Health

Evaluation 291 -, Mortality Prediction Model 291 Separationseffizienz 4237 Sepsis 2106

Serin/Threonin-Phosphatasen $1 / 2 \mathrm{~A}$

Suppl. 123 Serokonversion 4208 Serotonin Suppl. 123 Sicherheit von Blutprodukten 119

Singulettsauerstoff Suppl. 18 Skelettmuskel 2106 Solvent/Detergent Suppl. 12

Spenderausschluß Suppl. 189 Spezifisches IgE Suppl. 114 Stammzellapherese Suppl. 1116

Standardarbeitsanweisung 6364

Stoffwechsel 2 82,3 168 -, Fett 3168 -, Glukose 2 82,3 168 -, Insulin 282 -, Kalium 282 -, Leber 3168

Stufenplan, Nebenwirkungen von Blutkom-ponenten 3186

T-Antigen 4249 T-Zell-Depletion Suppl. 1119 Textile Implantate Suppl. 1165

Strukturen Suppl. 1165

-, Anwendungsgebiete Suppl. 1165

-, Eigenschaften Suppl. 1165 Thrombogenität Suppl. 1159 Thrombomatch EIA Suppl. 168

Thrombose Suppl. 163 Thrombozytapherese 4 237,4 244 Thrombozytäre Alloimmunisierung

Suppl. 177

- $\quad$ Antikörper Suppl. 168

Thrombozyten 6350 , Suppl. 123

Thrombozytenfunktion 5296

Thrombozyteninkrement 6350

Thrombozytenkonzentrate Suppl. 1 11,

Suppl. 1 14, Suppl. 123 Thrombozytenspender 114 -, bezahlte 114 -, Motivation 114 -, Zufriedenheit 114 Thrombozytensubstitution Suppl. 119 Thrombozytenzählung 5303 -, automatische 5303 -, mikroskopische 5303 Thrombozytopenie 5303 Tiefkühlkonservierung 3 152 Toxizität Suppl. 1159 Transfusion 6350 Transfusionsbedarf Suppl. 174

Transfusionstherapie 19 Transplantation Suppl. 1 116, Suppl. 1119 Tumorchirurgie 5280

Tumor-Nekrose-Faktor-alpha, TNF- $\alpha$

6332

Validierung Suppl. 12

Verzögerte hämolytische Transfusionsreak-

tion Suppl. 1101 Virusdekontamination Suppl. Ill Virusinaktivierung Suppl. 1 5, Suppl. 18

Virussicherheit Suppl. 12, Suppl. 195 Vollblut 5292

Wasserstoffionenkonzentration 298

Zellreduktion Suppl. 121 Zwerchfellgewebe 2106 Zytokine 5 292, Suppl. 114

388

Sachwortregister Band 22, 1995 